\title{
Über die Einwirkung von Pepsin auf Ovalbumin.
}

Von

R. O. Herzog und M. Margolis.

(Aus dem chemischen Institut der technischen Hochschule in Karlsruhe.)

(Der Redaktion zugegangen am 3. Mai 1909.)

Läßt man Pepsin auf Ovalbuminlösung wirken und mißt von Zeit zu Zeit die noch koagulable Menge des Proteins, dann findet man auch bei kleinen Fermentmengen sogleich nach der Vermischung der Lösungen eine sehr auffällige Abnahme der koagulierbaren Substanz. Im folgenden sei über das Ergebnis der Versuche kurz berichtet, die im Zusammenhang mit den quantitativen Fermentstudien des einen von uns im Sommer 1906 durchgeführt wurden.

Ovalbumin wurde in bekannter Weise ${ }^{1}$ ) hergestellt. Als Pepsin wurde das käufliche Präparat (Grübler) angewandt.

Zur quantitativen Bestimmung diente die Koagulation des gelösten Eiweißes und seine Wägung. Nach zahlreichen Vorversuchen wurde folgende Methodik eingehalten. Zur Koagulation wurde die Eiweißlösung in Reagenzgläsern von ca. $3 \mathrm{~cm}$ lichter Weite und etwa $23 \mathrm{~cm}$ Länge mit einem ihr gleichen Volumen gesättigter Natriumsulfatlösung und einigen Tropfen Salpetersäure versetzt und in ein siedendes Wasserbad gebracht. Der Niederschlag wurde dann zuerst dekantiert, mit Wasser gewaschen, bis im Filtrat keine Schwefelsäure mehr nachweisbar war, mit Alkohol nachgewaschen und 4 Stunden bei $120-130^{\circ}$ getrocknet; aus einer größeren Anzahl von Versuchen ging hervor, daß unter solchen Bedingungen Gewichtskonstanz eingetreten ist. Als Filtermaterial dienten die Filter Nr. 590 von Schleicher

1) Vgl. Langstein, Hofmeisters Beiträge, Bd. I, S. 97'(1902). 
und Schüll (durchschnittlicher Wassergehalt: $20 \mathrm{mg}$ ), die vor dem Versuch $3-4$ Stunden bei $120-130^{\circ}$ getrocknet wurden, eine Zeit, nach welcher Gewichtskonstanz eingetreten ist. Unter diesen Bedingungen beträgt der durchschnittliche Fehler einer Bestimmung bei Anwendung von etwa 0,5-0,05 g Eiwei $14 \mathrm{mg}$.

\section{Versuche.}

$\mathrm{Zu} 90 \mathrm{ccm}$ Flüssigkeit, die $0,462 \mathrm{~g}$ Ovalbumin und $0,36 \mathrm{~g}$ $\mathrm{HCl}$ enthält, werden $10 \mathrm{ccm}$ einer $3 \%$ igen Pepsinlösung gebracht. (Beide Lösungen sind, wie auch im folgenden stets, im Thermostaten vorgewärmt; die Reihenfolge der Vermischung ist stets die, daß zur Albuminlösung nacheinander Wasser und Salzsäure und schließlich das Ferment gebracht wird; Temperatur: $30^{\circ}$.)

Unmittelbar nach dem Vermischen sind nur mehr 74,5\% des Ovalbumins koagulierbar,

$$
\begin{aligned}
& \text { nach } 1 / 2 \text { Stunde } 70,8 \% \\
& \text { > } 21 / 2 \text { Stunden 58,7 * } \\
& \text { \ } 4^{1 / 2} \gg 52,9 \text { » } \\
& \text { - } 21 \text { ॰ } 42 \text {. }
\end{aligned}
$$

In einem parallelen Versuch werden gleich nach der Vermischung 76,9\% nach $1 / 2$ Stunde $68,8 \%$

$$
\begin{aligned}
& \text { ఎ }\left.2^{1}\right|_{2} \text { Stunden 57,2 * } \\
& \text { ^ } 41 / 2 \text { * } 55 \text {, }
\end{aligned}
$$

gefällt.

Weiterhin wird das Verhalten unter denselben Bedingungen geprüft nur mit dem Unterschied, daß in der Lösung diesmal $0,185 \mathrm{~g}$ Ovalbumin enthalten sind.

Sogleich nach dem Vermischen sind in einem Fall $63,2 \%$, im andern 71,9\% fällbar, nach 6 Stunden im ersten 89,7\%, im zweiten $85,9 \%$.

Zur Prüfung, ob etwa die Salzsäure bei der stattfindenden Veränderung Einfluß nimmt, werden $2 \mathrm{ccm}$ einer Ovalbuminlösung, die $0,185 \mathrm{~g}$ desselben enthalten, mit verschiedenen Mengen Salzsäure, Pepsin und Wasser in folgender Weise auf das Volumen von $10 \mathrm{ccm}$ gebracht und nach Vermischung der vorgewärmten Flüssigkeiten sogleich untersueht. 


\begin{tabular}{c|c|c|c|c|c}
\hline $\begin{array}{c}\text { Ovalbumin- } \\
\text { lösung } \\
\mathrm{ccm}\end{array}$ & $\begin{array}{c}\text { Normal- } \\
\mathrm{HCl} \\
\mathrm{ccm}\end{array}$ & $\begin{array}{c}\text { Pepsin- } \\
\text { lösung } \\
\mathrm{ccm}\end{array}$ & $\begin{array}{c}\text { Wasser } \\
\mathrm{ccm}\end{array}$ & $\begin{array}{c}\text { Gesamt- } \\
\text { volumen } \\
\mathrm{ccm}\end{array}$ & $\begin{array}{c}\text { Fallbares } \\
\text { Albumin } \\
\%\end{array}$ \\
\hline \multirow{2}{*}{} & 1 & 2 & 5 & 10 & 45 \\
2 & 1 & - & 7 & 10 & 96 \\
2 & - & 2 & 6 & 10 & 48,8
\end{tabular}

Ein anderer Versuch:

\begin{tabular}{c|c|c|c|c|c}
\hline $\begin{array}{c}\text { Ovalbumin- } \\
\text { lösung } \\
\text { ccm }\end{array}$ & $\begin{array}{c}\text { Normal- } \\
\text { HCl } \\
\mathrm{ccm}\end{array}$ & $\begin{array}{c}\text { Pepsin- } \\
\text { lösung } \\
\mathrm{ccm}\end{array}$ & Wasser & $\begin{array}{c}\text { Gesamt- } \\
\text { volumen } \\
\mathrm{ccm}\end{array}$ & $\begin{array}{c}\text { Fällbares } \\
\text { Albumin } \\
\%\end{array}$ \\
\hline 2 & 1 & 2 & 5 & 10 & 47,1 \\
2 & 1 & - & 7 & 10 & 100
\end{tabular}

Es zeigt sich also, daß nicht die Säure die Abnahme an fällbarer Substanz bewirkt, sondern das Ferment.

Die weiteren Versuche sind zur Verringerung der Reaktionsgeschwindigkeit mit verdünnteren Pepsinlösungen $(0,6$ und $1 \%$ ig), ferner nur mit einem Salzsäuregehalt von $1 / 20$-normal (bisher 0,1 normal) und bei $0^{\circ}$ durchgeführt.

Die Abhängigkeit von der Fermentmenge geht aus folgenden Zahlen hervor. (Die Zeit zwischen Vermischung der sauren Lösung mit dem Ferment und der Fällung beträgt 2 Minuten, hier wie künftig mit einer Rennuhr bestimmt.)

\begin{tabular}{c|c|c|c|c|c}
\hline $\begin{array}{c}\text { Ovalbuminlösung } \\
0,185 \text { g enthaltend } \\
\mathrm{ccm}\end{array}$ & $\begin{array}{c}\text { Normal- } \\
\mathrm{HCl} \\
\mathrm{ccm}\end{array}$ & $\begin{array}{c}\text { Pepsinlösung } \\
0,6 \% \text { ig } \\
\mathrm{ccm}\end{array}$ & $\begin{array}{c}\text { Wasser } \\
\mathrm{ccm}\end{array}$ & $\begin{array}{c}\text { Gesamt- } \\
\text { volumen } \\
\mathrm{ccm}\end{array}$ & $\begin{array}{c}\text { Fällbares } \\
\text { Albumin } \\
\%\end{array}$ \\
\hline & 0,5 & 0,5 & 7 & 10 & 87,6 \\
2 & 0,5 & 1 & 6,5 & 10 & 82,7 \\
2 & 0,5 & 2 & 5,5 & 10 & 77,3
\end{tabular}

Dasselbe Ergebnis zeigt der nachstehende Versuch.

Die Zeit zwischen Vermischung und Fällung beträgt 10 Minuten. 
Über die Einwirkung von Pepsin auf Ovalbumin.

\begin{tabular}{c|c|c|c|c|c}
\hline $\begin{array}{c}\text { Ovalbuminlösung } \\
\mathbf{0 , 1 8 5} \mathrm{g} \\
\mathrm{ccm}\end{array}$ & $\begin{array}{c}\text { Normal- } \\
\mathrm{HCl} \\
\mathrm{ccm}\end{array}$ & $\begin{array}{c}\text { Pepsinlösung } \\
1 \% \mathrm{ig} \\
\mathrm{ccm}\end{array}$ & Wasser & $\begin{array}{c}\text { Gesamt- } \\
\text { volumen } \\
\mathrm{ccm}\end{array}$ & $\begin{array}{c}\text { Fällbares } \\
\text { Ovalbumin } \\
\% \%\end{array}$ \\
\hline 2 & 0,5 & 0,1 & 7,4 & 10 & 92 \\
2 & 0,5 & 0,4 & 7,1 & 10 & 84,4 \\
2 & 0,5 & 0,9 & 6,4 & 10 & 77,3 \\
2 & 0,5 & 1,5 & 6 & 10 & 73 \\
2 & 0,5 & 2,5 & 5 & 10 & 66
\end{tabular}

Endlich sei noch eine Versuchsreihe angeführt, bei welcher keine Salzsäure zugesetzt ist. Die ursprünglich vorhandene Menge 0valbumin beträgt $0,185 \mathrm{~g}$, das Gesamtvolumen $10 \mathrm{ccm}$, die Zeit zwischen Vermischung und Fällung 15 Minuten; Temperatur: $0^{\circ}$.

\begin{tabular}{c|c}
\hline $\begin{array}{c}1 \% \text { ige Pepsinlösung } \\
\text { ccm }\end{array}$ & $\begin{array}{c}\text { Fällbares Ovalbumin } \\
\%\end{array}$ \\
\hline 0,1 & 95,2 \\
0,4 & 88,7 \\
0,9 & 83,3 \\
1,6 & 76,2 \\
2,5 & 74,1
\end{tabular}

Berechnet man die Geschwindigkeit $\mathbf{v}$ der 3 angegebenen Versuchsreihen wie für eine Reaktion erster Ordnung (ohne Berücksichtigung der Zeiten, die jedesmal konstant sind) und vergleicht sie mit dem Wert, der nach der bekannten Schütz-

\begin{tabular}{|c|c|c|c|c|c|}
\hline \multicolumn{2}{|c|}{ 1. Versuch, $\mathrm{k}=0,057$} & \multicolumn{2}{|c|}{ 2. Versuch, $\mathrm{k}=0,036$} & \multicolumn{2}{|c|}{ 3. Versuch, $\mathrm{k}=0,026$} \\
\hline $\begin{array}{c}\mathbf{v} \\
\text { gefunden }\end{array}$ & $\begin{array}{c}\mathbf{v} \\
\text { berechnet }\end{array}$ & $\stackrel{\mathbf{v}}{\text { gefunden }}$ & $\begin{array}{c}\mathbf{v} \\
\text { berechnet }\end{array}$ & $\begin{array}{c}\mathbf{v} \\
\text { gefunden }\end{array}$ & $\begin{array}{c}\mathbf{v} \\
\text { berechnet }\end{array}$ \\
\hline 0,058 & 0,057 & 0,037 & 0,036 & 0,022 & 0,026 \\
\hline 0,082 & 0,082 & 0,074 & 0‘072 & 0,052 & 0,052 \\
\hline 0,112 & 0,114 & 0,112 & 0,108 & 0,080 & 0,078 \\
\hline- & - & 0,137 & 0,144 & 0,118 & 0,104 \\
\hline - & 一 & 0,181 & 0,180 & 0,130 & 0,130 \\
\hline
\end{tabular}


Borissowschen Regel für Pepsin $v=k \sqrt{\mathrm{f}}$ (f die relative Fermentmenge, die schwächste Konzentration $=1$ gesetzt; $\mathrm{k}$ eine Konstante) erhalten wird, so zeigt sich gute Übereinstimmung, wie die vorstehende Tabelle lehrt.

Die nächste Versuchsreihe ist mit konstanter Fermentmenge (1 ccm einer 1\% \%igen Lösung) und variabeln Ovalbuminmengen bei dem Volumen $10 \mathrm{ccm}$ und ohne Salzsäurezusatz durchgeführt. Zeit zwischen Fermentzusatz und Fällung: 1 Stunde.

\begin{tabular}{c|c|c|c}
\hline \hline Ovalbumin & $\begin{array}{c}\text { Fällbares } \\
\text { Ovalbumin } \\
\text { in } \mathbf{g}\end{array}$ & $\begin{array}{c}\text { Nicht fällbar } \\
\text { in } \mathrm{g}\end{array}$ & $\begin{array}{c}\text { Reaktions- } \\
\text { geschwindigkeit }\end{array}$ \\
\hline 0,092 & 0,051 & 0,041 & 0,256 \\
0,185 & 0,147 & 0,038 & 0,100 \\
0,277 & 0,236 & 0,041 & 0,070 \\
0,370 & 0,332 & 0,038 & 0,047 \\
0,462 & 0,432 & 0,030 & 0,029
\end{tabular}

Es zeigt sich, daß die Regel, je konzentrierter das Substrat, desto kleiner die Reaktionsgeschwindigkeit, ${ }^{1}$ ) hier wie sonst bei den Fermentreaktionen zutrifft, auch die Regel von Brown und Armstrong, ${ }^{2}$ ) daß in gleichen Zeiten gleichviel umgesetzt wird, erscheint annähernd bestätigt.

Zum Vergleich wurden auch einige Versuche mit käuflichem Lab gemacht. Um eine merkbare proteolytische Wirkung zu erzielen, mußte das Präparat längere Zeit bei $30^{\circ}$ digeriert werden. Von dieser klar filtrierten Lösung wurden in einem Versuch $2 \mathrm{ccm}$ zu $10 \mathrm{ccm}$ Ovalbuminlösung (enthaltend: $0,925 \mathrm{~g}$ ), $5 \mathrm{ccm}$ Normal-HCl und $33 \mathrm{ccm}$ Wasser gebracht (Gesamtvolumen $=50 \mathrm{ccm}$ ), in einem zweiten Versuch $5 \mathrm{ccm}$ des Ferments zu $10 \mathrm{ccm}$ Ovalbumin, $5 \mathrm{ccm}$ Normal-HCl und $30 \mathrm{ccm}$ Wasser (Gesamtvolumen $=50 \mathrm{ccm}$ ). Temperatur: $30^{\circ}$.

1) R. O. Herzog, Diese Zeitschrift, Bd. XLVIII, S. 366 (1906).

2) Vgl. Brown, J. chem. Soc., Bd. LXI, S. 380 (1892), Proc. Chem. Soc., Bd. XVIII, S. 41 (1902), u. a. a. 0. 


\begin{tabular}{|c|c|c|c|c|}
\hline \multirow{2}{*}{$\begin{array}{l}\text { Nach } \\
\text { Tagen }\end{array}$} & \multicolumn{2}{|c|}{$2 \mathrm{ccm}$ Ferment } & \multicolumn{2}{|c|}{$5 \mathrm{ccm}$ Ferment } \\
\hline & $\begin{array}{c}\text { Fällbares } \\
\text { Albumin } \\
\text { in } \%\end{array}$ & $\begin{array}{l}\text { Reaktions- } \\
\text { geschwindig- } \\
\text { keit }\end{array}$ & $\begin{array}{c}\text { Fällbares } \\
\text { Albumin } \\
\text { in } \%\end{array}$ & $\begin{array}{l}\text { Reaktions- } \\
\text { geschwindig- } \\
\text { keit }\end{array}$ \\
\hline 1 & 77,3 & 0,112 & 69,2 & 0,160 \\
\hline 2 & 73,6 & 0,067 & 62,7 & 0,101 \\
\hline 3 & 70,3 & 0,051 & 60,1 & 0,074 \\
\hline 4 & 66 & 0,045 & 52,3 & 0,070 \\
\hline
\end{tabular}

Aus der auf S. 302 gegebenen Form der Schützschen Regel folgt, daß für gleiche Zeiten $\frac{v}{\sqrt{\mathrm{f}}}=\mathrm{k}$ sein muß.

Die folgende Tabelle enthält die aus den angeführten Versuchen berechneten Werte von $\mathbf{k}$, die also für gleiche Zeiten bei verschiedenen Fermentmengen konstant sein sollen.

\begin{tabular}{c|c|c}
\hline Tage & 2 ccm. Ferment & 5 ccm Ferment \\
\hline 1 & 0,079 & 0,073 \\
2 & 0,047 & 0,045 \\
3 & 0,036 & 0,033 \\
4 & 0,032 & 0,031
\end{tabular}

Der Wert von $k$ ist für die größere Fermentkonzentration stets etwas geringer als für die kleinere; dies Verhalten tritt im allgemeinen dann bei Fermentreaktionen ein, wenn die absolute Fermentkonzentration sehr groß ist. Daß dieser Fall hier wirklich vorliegt, obwohl die Reaktion sich so langsam abspielt, wird auch noch durch folgenden Versuch wahrscheinlich gemacht.

Die Fermentlösung ist $10 \mathrm{mal}$ so konzentriert wie bei den vorangegangenen Versuchsreihen; die Temperatur beträgt $30^{\circ}$. Bei der ersten Fällung liegen zwischen Vermischung und Fällung 3 Stunden, bei der zweiten 14 Stunden. 
R. 0. Herzog und M. Margolis,

\begin{tabular}{|c|c|c|c|c|c|}
\hline $\begin{array}{c}\text { Ovalbumin } \\
0,185 \mathrm{~g} \\
\mathrm{ccm}\end{array}$ & $\begin{array}{c}\text { Normal- } \\
\mathrm{HCl} \\
\mathrm{ccm}\end{array}$ & $\begin{array}{c}\text { Wasser } \\
\text { ccm }\end{array}$ & $\begin{array}{l}\text { Lab- } \\
\text { lösung } \\
\text { ccm }\end{array}$ & $\begin{array}{c}\text { Nach } \\
3 \text { Stunden. } \\
\text { Fällbares } \\
\text { Albumin } \\
\%\end{array}$ & $\begin{array}{c}\text { Nach } \\
14 \text { Stunden. } \\
\text { Fällbares } \\
\text { Albumin } \\
0 \% 9\end{array}$ \\
\hline 2 & 1 & 6 & 1 & 82,3 & 66 \\
\hline 2 & 1 & 5 & 2 & 81,2 & 64 \\
\hline 2 & 1 & 3 & 4 & 83,3 & 66 \\
\hline 2 & 1 & 7 & 0 & - & 100 \\
\hline
\end{tabular}

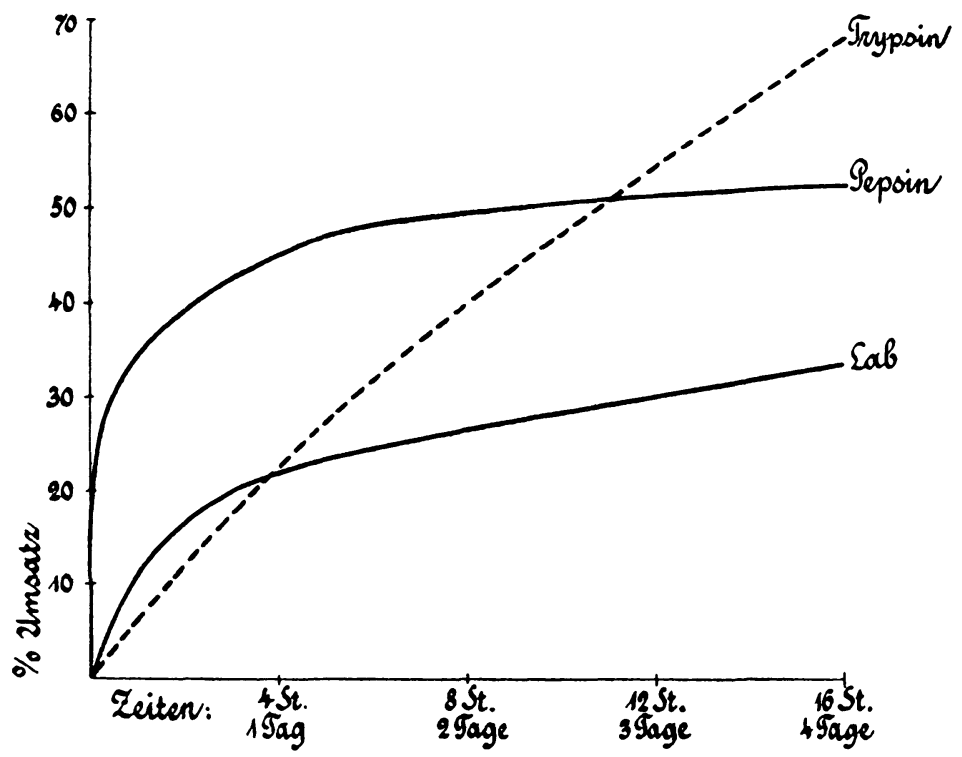

(Die Stunden beziehen sich auf die Pepsin-, die Tage auf die Lab- und Trypsinwirkung.)

Man sieht, daß bei dieser Stärke der Fermentlösung ihre Konzentration ohne Einfluß auf die Geschwindigkeit ist; wie das eben nur bei höchst konzentrierten Fermentlösungen gilt. Die Versuche machen es außerordentlich wahrscheinlich, daß in dem Labpräparat die proteolytische Wirkung in der Weise gehemmt ist, daß zwar die absolute Geschwin- 
digkeit außerordentlich herabgesetzt ist, daß aber die Relation zwischen Fermentkonzentration und Reaktionsgeschwindigkeit nicht gestört wird. Die vorstehenden Umsatzkurven zeigen auch, daß der Reaktionsverlauf bei dem Pepsin- und Labpräparat sehr ähnlich ist; die zum Vergleich eingezeichnete Trypsinwirkung gibt ein ganz anderes Bild. ${ }^{1}$ )

Aus den mitgeteilten Versuchen geht hervor,

1. daß bereits mäßig starke Pepsinlösungen sogleich nach dem Vermischen mit Ovalbumin einen erheblichen Anteil desselben der Hitzekoagulation entziehen und diese Fermentwirkung der Schütz-Borissow schen Regel entspricht, somit ohne Zweifel als Pepsinwirkung anzusprechen ist;

2. daß Lablösungen sich dem Ovalbumin gegenüber in jeder Beziehung ebenso verhalten wie Pepsinlösungen und der Unterschied ihrer Wirkung nur in einer erheblichen Dämpfung der Reaktionsgeschwindigkeit liegt.

1) Die Fällungen sind nach Pepsinwirkung großlockig, bei Anwendung von Lab nahezu gallertartig, von Trypsin feinflockig. Für die Trypsinpräparate findet man nicht die auf S. 302 angeführte Beziehung. (Schützsche Regel), sondern $\mathbf{v}=\mathbf{k} \cdot \mathbf{f}$. 